\title{
Representaciones subjetivas y competencias cognitivas en el aprendizaje de una nueva pieza de música contemporánea para piano*
}

SUBJECTIVE REPRESENTATIONS AND COGNITIVE COMPETENCIES IN THE LEARNING
PROCESS OFA NEW CONTEMPORARY MUSIC PIECE FOR PIANO

REPRESENTAÇÕES SUBJETIVAS E HABILIDADES COGNITIVAS NA APRENDIZAGEM

DE UMA NOVA PEÇA DE MÚSICA CONTEMPORÂNEA PARA PIANO

\section{Michel A. Cara**}

Cuadernos de Música, Artes Visuales y Artes Escénicas

/ Volumen 13- Número 2 / Julio - Diciembre de 2018

/ ISSN 1794-6670/ Bogotá, D.C., Colombia / pp. 205-226

Fecha de recepción: 15 de julio de 2016

Fecha de aceptación: 13 de junio de 2017

Disponible en línea: 1 de junio 2018

doi:10.11144/javeriana.mavae13-2.rsyc

* $\quad$ Artículo de investigación realizado en el marco de una tesis de doctorado financiada por la Comisión Nacional de Investigación Científica y Tecnológica (CONICYT), folio n. ${ }^{\circ} 72100347$.

* Licenciado en Artes con mención en Teoría de la Música por la Universidad de Chile, Magíster en Análisis de Sistemas Educativos; Magister en Psicología Cognitiva, Desarrollo y Psicopatología Clínica; y doctor en Ciencias de la Educación, todos por la Universidad de Borgoña. Profesor de Educación Musical de la Pontificia Universidad Católica de Chile. ORCID: 0000-0001-8321-700X 


\section{Resumen}

El proceso de aprendizaje de una nueva pieza de música contemporánea para piano fue estudiado en un grupo de diez estudiantes de piano funcional, todos guiados por la profesora Kenya Godoy, docente de la Facultad de Artes de la Universidad de Chile. El objetivo de la investigación es analizar las distintas estrategias de estudio de los estudiantes en función de sus capacidades cognitivas considerando la interacción educativa como parte relevante del fenómeno de aprendizaje. Los participantes leyeron cuatro veces la pieza Algunas preguntas del compositor chileno Juan Amenábar, con la posibilidad de dos minutos de práctica entre cada interpretación, sin detención, de la obra musical completa. Se aplicaron cuestionarios a los estudiantes y a la profesora, así como pruebas de memoria operativa y espacial a corto plazo a los estudiantes. Lo anterior con la finalidad de analizar la implicancia de los recursos cognitivos en el aprendizaje musical y el tipo de representación mental involucrada en la performance. La profesora se sitúa fuera del proceso de aprendizaje, pero participa posteriormente analizando las interpretaciones de sus estudiantes registradas en formato video. El análisis de los cuestionarios demuestra que la eficacia personal se refleja en el grado de confianza que el profesor entrega a su alumno y establece una relación dialéctica que refuerza la integración del conocimiento a largo plazo. Un análisis de componentes principales muestra que la evaluación del profesor parece ser un compromiso entre las capacidades cognitivas del estudiante, sostenidas por la confianza personal y el desempeño real guiado por los aspectos técnicos.

Palabras clave: aprendizaje musical; lectura musical; música contemporánea; interacción profesor-estudiante; autoeficacia; evaluación musical.

\section{Abstract}

The learning process of a new piece of contemporary music for piano was studied in a group of ten functional piano students, all guided by instructor Kenya Godoy, faculty member from the School of Arts at Universidad de Chile. The goal of the research is to analyze the different study strategies of students based on their cognitive capacities, considering the educational interaction as a relevant part of the learning phenomenon. The participants read the piece Algunas preguntas from Chilean composer Juan Amenábar four times, with the possibility of a two-minute practice between each interpretation, without pause, of the entire music piece. Questionnaires were applied to students and to the professor, as well as other operational and spatial short-term memory tests applied to students. This was with the purpose of analyzing the implication of cognitive resources in the process of learning musical and the type of mental representation involved in the performance. The instructor is placed outside of the learning process, but she participates afterwards by analyzing the interpretations of her students registered in video format. The analysis of the questionnaires shows that self-efficacy is reflected in the degree of trust that the instructor gives his student and established a dialectical relationship that reinforces the long-term integration of knowledge. A principal components analysis shows that the instructor's assessment seems to be a commitment between the cognitive capacities of the student, supported by personal trust and real performance and guided by the technical aspects.

Keywords: music learning process; music reading; contemporary music; instructor-student interaction; self-efficacy; musical assessment.

\section{Resumo}

O processo de aprendizagem de uma nova peça de música contemporânea para piano foi estudado em um grupo de dez estudantes de piano funcional, todos guiados pela professora Kenya Godoy, docente da Faculdade de Artes da Universidade do Chile. O objetivo da pesquisa é analisar as diferentes estratégias de estudo dos estudantes em função de suas capacidades cognitivas considerando a interação educativa como parte relevante do fenômeno de aprendizagem. Os participantes leram quatro vezes a peça Algunas preguntas -(Algumas perguntas) do compositor chileno Juan Amenábar, com a possibilidade de dois minutos de prática entre cada interpretação, sem detenção, da obra musical completa. Aplicaram-se questionários aos estudantes e a professora, assim como provas de memória operativa e espacial a curto prazo aos estudantes. O supra citado com a finalidade de analisar a implicação dos recursos cognitivos na aprendizagem musical e no tipo de representação mental envolvida na performance. A professora se situa fora do processo de aprendizagem, mas participa posteriormente analisando as interpretações de seus estudantes registradas em formato vídeo. A análise dos questionários demonstra que a eficácia pessoal se reflete no grau de confiança que o professor entrega ao seu aluno e estabelece uma relação dialética que reforça a integração do conhecimento a longo prazo. Uma análise de componentes principais mostra que a avaliação do professor parece ser um compromisso entre as capacidades cognitivas do estudante, sustentadas pela confiança pessoal e o desempenho real guiado pelos aspectos técnicos.

Palavras-chave: aprendizagem musical; leitura musical; música contemporânea; interação professor-estudante; auto eficácia; avaliação musical. 


\section{INTRODUCCIÓN}

En este trabajo, analizaremos desde un enfoque mixto (i. e. cualitativo y cuantitativo) cómo se produce el aprendizaje de una pieza de música contemporánea en una clase de piano. El objetivo responde a la necesidad de desarrollar una estrategia educativa que ayude al músico en su formación. Nos interesa estudiar el fenómeno del aprendizaje en su contexto, es decir, en una misma clase de piano utilizando repertorio contemporáneo latinoamericano. Examinaremos el proceso de aprendizaje y su relación con el desarrollo de las competencias cognitivas de los músicos como las concepciones o representaciones subjetivas que tienen de su propio proceso de aprendizaje. Cabe señalar que los modelos actuales en este campo de estudio se han referido, principalmente, a la música tonal y han sido establecidos en su mayoría sobre la base de extractos de piezas musicales, lo cual limita el acceso a la estructura general de la pieza. Por esta razón, creemos que es pertinente abordar en el presente estudio una pieza completa de música contemporánea. ${ }^{1}$

\section{COMPETENCIAS MUSICALES Y APRENDIZAJE}

La lectura musical es una competencia que necesita muchas horas de práctica para adquirir fluidez y rapidez. Desde un punto de vista cognitivo, es una tarea de transcripción compleja que involucra una serie de procesos perceptivos y psicomotores (Sloboda 1982). En efecto, según Ericsson, Krampe y Tesch-Römer (1993), la representación funcional de la música escrita es construida en relación con conocimientos específicos, lo cual podría involucrar procesos de neuroplasticidad tanto estructurales como funcionales ${ }^{2}$ que están específicamente ligados a la performance musical.

Dentro de las competencias cognitivas específicas relacionadas con la música escrita, se destaca la lectura a primera vista, definida por Wolf (1976) como la capacidad de leer una partitura por primera vez sin el beneficio de la práctica. Con la finalidad de estudiar las distintas variables que inciden en la lectura a primera vista, Kopiez y Lee (2008) desarrollan un modelo de múltiples componentes basado en competencias cognitivas generales ( $p$. ej. memoria operativa), elementares (p. ej. velocidad de procesamiento de la información) y aquellas competencias relacionadas con la práctica (p. ej. oído interno). Los autores muestran que la lectura a primera vista puede predecirse, por una parte, según una combinación entre variables relacionadas con la práctica musical (i. e. velocidad psicomotora, experticia musical adquirida tempranamente y la habilidad para generar imágenes auditivas); y por otra, por variables determinadas genéticamente como la velocidad mental.

Desde esta perspectiva, nos hemos propuesto estudiar el proceso de aprendizaje de una nueva pieza de música contemporánea desde distintos niveles: 1) recursos cognitivos, 2) estrategias de procesamiento, toma y recuperación de la información y 3) proceso de evaluación/retroalimentación.

Pensamos que el proceso metodológico necesario para abordar una nueva pieza de música contemporánea podría diferenciarse de aquel necesario para abordar una pieza de música clásico-romántica. En primer lugar, desde el punto de vista de las estrategias de procesamiento de la información, los procesos de agrupamiento o segmentación en unidades musicales se llevarían a cabo según estructuras diferentes de las estructuras tonales. 
En efecto, tal como lo plantea McAdams (1989), muchas obras contemporáneas (desde la década de 1950) han sido elaboradas siguiendo un principio estético que evita el llamado a aquellos esquemas característicos de la música clásico-romántica, con lo cual fuerza (con estas irregularidades) a que los auditores tengan una forma completamente distinta de escuchar y recordar la música (187). Según el autor, toda la información musical es interpretada tomando como base estructuras de conocimiento previamente adquiridas. Por tanto, los procesos de agrupamiento tienen un efecto en la percepción de la forma musical y actúan sobre las cualidades emergentes del discurso musical (p. ej. ritmo, consonancia, timbre).

En segundo lugar, desde el punto de vista de las estrategias de toma de información, los mecanismos de selección de la información relevante podrían operar de manera dinámica de acuerdo con los diferentes tipos de notación e involucrar diferentes habilidades perceptivas. Al respecto, Cara y Gómez (2016) estudian en un grupo de estudiantes de piano funcional el comportamiento oculomotor durante una tarea de lectura silenciosa de breves piezas en estilos contrastantes (tonal vs. contemporáneo). Se analiza el número y la duración de las fijaciones oculares, tanto a nivel intrafrase como a nivel interfrase. Los resultados muestran que, en la música contemporánea, el número de fijaciones regresivas interfrase (necesarias para los procesos de integración de la información) es más frecuente, particularmente en la fase de relectura, en relación con la música tonal. Lo anterior sugiere el uso de distintas estrategias de selección y de procesamiento de la información según el estilo musical e indica la presencia de inspecciones menos secuenciales en la música contemporánea.

En tercer lugar, la generación de expectativas musicales se relaciona con las estrategias de recuperación de la información. En efecto, tanto la violación como la confirmación de las expectativas influyen en la experiencia estética, en la construcción de sentido y en la comunicación de las emociones (Meyer 1956; Narmour 1990; Pearce y Wiggins 2006). Desde el punto de vista del modelo de memoria operativa a largo plazo ${ }^{3}$ de Ericsson y Kintsch (1995), los músicos expertos serían capaces de elaborar estructuras de recuperación de la información, que no solo les permitiría recordar, sino también preparar o anticipar los eventos musicales, lo cual sugiere que las expectativas musicales están sujetas al tipo de estilo musical y, por tanto, a la experiencia musical previa. En efecto, Cara (2017), en un estudio conducido con 22 pianistas profesionales, muestra que el aprendizaje a corto plazo de una nueva pieza de música contemporánea está asociada con la movilización de competencias visoperceptivas, lo cual permite aumentar las capacidades de anticipación (eye-hand span o distancia entre el punto de fijación ocular y la nota ejecutada). Finalmente, en el proceso de retroalimentación, el rol del profesor podría ser esencial, particularmente en las fases iniciales de familiarización con la lectura de la música contemporánea, lo que exploraremos más en detalle en el presente estudio.

En síntesis, la lectura musical, y particularmente el desarrollo de las capacidades de lectura a primera vista, depende de distintos factores tanto externos (p. ej. contexto de aprendizaje, tipo de estímulo, tipo de tarea) como internos (p. ej. experticia, capacidades cognitivas, estrategias de aprendizaje). Desde un punto de vista metodológico, pensamos que el profesor debe conocer las capacidades de sus estudiantes, así como sus expectativas y motivaciones. A partir de nuestros trabajos previos (Cara, 2016, 2017), podemos señalar que cada estilo musical requiere el desarrollo de competencias específicas y, por tanto, podría solicitar determinados recursos cognitivos según el tipo de material musical. 
Desde esta perspectiva, tanto en la partitura de la música clásica como de la romántica, las estrategias metodológicas podrían apuntar hacia la adquisición de cierta independencia del código escrito (capacidad del músico de elaborar una representación completa de la partitura a partir de ciertos índices), el desarrollo de estrategias de selectividad de la información relevante (procesos de agrupamiento) y el manejo de información de distinta fuente (competencia intermodal), con un énfasis en la comprensión de las particularidades propias de cada compositor, en muchas obras románticas. En la música contemporánea, el proceso metodológico podría apuntar hacia el desarrollo de competencias específicas como la atención dividida, la conciencia corporal, el manejo del gesto corporal, la improvisación o el manejo de técnicas de notación musical propias de cada compositor/escuela.

A pesar de los esfuerzos realizados en trabajos anteriores por definir las principales variables que influyen en el desarrollo de la lectura a primera vista (Kopiez y Lee 2008), actualmente no existe ningún modelo estable que logre dar cuenta de manera satisfactoria de la compleja dinámica que existe entre los factores que determinan las performances en lectura a primera vista. Lo anterior tanto en las diferencias interindividuales como en el material musical (p. ej. entre distintos estilos musicales). Es necesario, entonces, comenzar a observar el fenómeno paso a paso, por ejemplo, mediante observaciones microgenéticas, que es lo que haremos, precisamente, en el presente estudio, extendiéndonos desde una primera lectura hacia fases posteriores del aprendizaje.

Dados estos antecedentes, en este marco, analizaremos las fases iniciales del aprendizaje, es decir, desde una primera lectura o lectura a primera vista, hasta completar cuatro ejecuciones de la misma pieza. Como lo hemos mencionado, nos interesa el aprendizaje en su contexto, lo cual nos lleva a abordar el concepto de interacción educativa.

\section{APRENDIZAJE E INTERACCIÓN}

La experiencia, el contexto y las limitaciones propias del sistema cognitivo determinan la manera de aprender de un individuo. Esta dimensión dinámica del aprendizaje se expresa, desde la perspectiva de Maturana $(2002,241)$, como un proceso de transformación en la convivialidad. Se puede, entonces, convivir cuando se respeta al otro y se expresa esta confianza en un cambio de comportamiento que toma forma en la interacción. Desde un punto de vista educativo, la relación de confianza entre el profesor y el alumno forma parte de la dimensión afectiva del aprendizaje y se construye gracias al mérito, el respeto y la reciprocidad.

Para los fines del presente estudio, delinearemos la interacción desde una perspectiva espacio-temporal, ya que la naturaleza de esta última puede ser muy vasta. En efecto, según Bressoux y Dessus (2003), a pesar de que la interacción designaría la actividad del profesor frente a una clase, podrían existir distintas posibilidades de estudiar el fenómeno. Desde esta perspectiva, la naturaleza de la interacción podría ser oral o bien escrita y podrían presentarse dificultades para definir quiénes realizan, en efecto, la interacción. ¿Solo profesores y estudiantes?

Nos limitaremos, por ende, a analizar la interacción entre el profesor y el alumno tomando como base verbalizaciones y cuestionarios retrospectivos. O'Sullivan et al. (1997) entienden la interacción como "el intercambio y la negociación del significado entre dos o más participantes en determinados contextos sociales" (196). Esta construcción de significado, desde un punto 
de vista pedagógico, ocurre en situaciones de aprendizaje donde la relación de interacción se constituye en dinámicas compartidas, reglas y normas establecidas como parte de un proceso adaptativo (Rizo 2006). El autor subraya la importancia de la comunicación interpersonal como elemento base de la comunicación humana, ya que permite el intercambio de subjetividades y la construcción de sentido.

En efecto, parte de la dimensión social del aprendizaje consiste en la construcción de sentido, a través de un proceso de transformación, que es regulado desde un punto de vista pedagógico por la evaluación. Lo anterior nos abre las puertas hacia el componente cualitativo de la interacción, donde la cooperación entre el profesor y el alumno puede convertirse, tal como lo demuestra Bogunovic (2003), en un factor de éxito para los estudiantes que inician sus estudios musicales. El autor logra identificar en un estudio longitudinal realizado en cinco escuelas especializadas en música en Belgrado distintos factores sociopsicológicos que inciden en el logro académico y performativo (conciertos y concursos). No obstante, la adquisición de competencias en los estudiantes dotados depende, según el autor, preferentemente del nivel de preparación del profesor.

\section{RECURSOS COGNITIVOS}

Durante el proceso de aprendizaje, se requiere la capacidad de la memoria operativa, modelo teórico introducido por Baddeley y Hitch (1974), y que posteriormente ha sido reformulado (Baddeley 2012). Este sistema se encarga de almacenar temporalmente la información mientras es procesada, y permite, en la lectura musical, enriquecer la información nueva con la anteriormente procesada, de manera de dar coherencia al discurso musical (Servant y Baccino 1999). Dichos mecanismos integrativos permiten modificar de manera constante las representaciones mentales durante la lectura. La memoria operativa podría entenderse entonces como un lugar de transformación de la información (68). La capacidad de la memoria operativa puede ser determinante en la adquisición de competencias musicales, particularmente en la lectura de partituras, tal como lo planeta Berz (1995). En efecto, el autor sugiere que existe una relación entre la memoria operativa y el éxito en las pruebas de aptitud musical, por ejemplo en aquellos ítems que implican recordar y comparar extractos musicales.

En lo particular, las capacidades de la memoria visoespacial serían solicitadas durante la lectura de partituras, dada su naturaleza espacial. Stewart et al. (2003), utilizando una tarea de stroop musical, compara pianistas con no músicos, demostrando que los pianistas desarrollan mapeos verticales a horizontales que se pueden utilizar independiente del contexto musical. Por su parte, Sergent et al. (1992) realizan un estudio por medio de técnicas de resonancia magnética y de tomografía por emisión de positrones con diez pianistas profesionales. Los autores muestran que el lóbulo parietal superior se activaría con la naturaleza espacial de la notación musical (durante una tarea de lectura a primera vista), lo cual indica que la música y la información verbal comparten áreas cerebrales adyacentes, pero funcionalmente diferentes. Utilizando la técnica de mapeo cerebral directo, Roux et al. (2007) muestran que el lóbulo parietal inferior izquierdo se activa durante la lectura de la música. Según los autores, la corriente visual dorsal, responsable del procesamiento visoespacial, está vinculada a la activación de los lóbulos parietales durante la lectura de la música.

Por lo demás, existe evidencia de que los músicos tienen mejores capacidades visoespaciales que los no músicos. Al respecto, Brochard, Dufour y Després (2004) muestran que los músicos tienen mejor percepción, especialmente en la dimensión vertical en comparación con la horizontal 
(interacción entre la experiencia y la discriminación de los ejes). Los autores sugieren que los tiempos de reacción son más eficaces en los músicos, debido a la adquisición de una tasa de asociación superior entre un estímulo visual y la respuesta motora. Esta premisa fue confirmada por Bo y Seidler (2009), para quienes el aprendizaje secuencial motor está relacionado con la capacidad de la memoria visoespacial. Los autores encontraron correlaciones positivas entre la capacidad de la memoria operativa, la tasa de aprendizaje temprano y la longitud de las agrupaciones o chunks realizadas por los participantes, en una tarea de aprendizaje secuencial explícito. Por su parte, los resultados de Anguera et al. (2010) confirman, igualmente, el compromiso de la memoria espacial en el aprendizaje visomotor. Lo anterior subraya la importancia de la memoria operativa y de la memoria espacial a corto plazo en la adquisición de competencias en lectura musical.

\section{OBJETIVOS DEL ESTUDIO}

Con el fin de considerar el aprendizaje como un proceso, estudiaremos 1) cómo se produce la adquisición de competencias musicales en lectura, en función de los recursos cognitivos de los pianistas; 2) la calidad de la interacción profesor-alumno en el proceso de enseñanza-aprendizaje; 3) las representaciones subjetivas que elaboran los pianistas sobre su propia forma de aprender; 4) las metodologías de enseñanza y las estrategias de aprendizaje asociadas al estudio de una pieza de música contemporánea; y 5) las distintas modalidades (i. e. visual, cinestésica, auditiva u otras) comprometidas en la performance.

Es necesario para este fin considerar los aspectos externos (es decir, qué aprenden y cómo aprenden) y las "interpretaciones subjetivas que los estudiantes construyen" en su aprendizaje (Coll 1988, 140). Según Marchand (2009), durante el proceso de aprendizaje, se generan dificultades que producen tensión y que pueden expresarse verbalmente. El autor, inspirándose en las concepciones de Lev Vygotsky y Jean Piaget en lo que respecta a la toma de consciencia en la acción, describe el proceso de tensión-dinámica generado como producto de la interacción entre la verbalización y la acción musical. Desde esta perspectiva, la competencia se desarrolla gracias a una fusión entre la toma de conciencia y la acción, lo cual permite, al mismo tiempo, comprender la regulación y la evaluación como un proceso de comunicación (Genthon 1997, 61-62).

De esta manera, las construcciones subjetivas de los estudiantes son un referente del tejido complejo de interacciones educativas presentes en el currículo (Covarrubias y Martínez 2007), lo cual permitiría generar cambios en las prácticas educativas. Estas construcciones operan como puente (mediador) entre la relación del músico consigo mismo y con su entorno, permitiendo, según Avendaño, Krause y Winkler (1993), que los individuos: 1) fundamenten su propia manera de actuar ante ellos mismos, 2) justifiquen los propios problemas tanto psicológicos como de otra índole, 3) orienten su comportamiento, 4) desarrollen la identidad, 5) den un sentido a la existencia (110) y 6) sean capaces de negociar con sus propias creencias y las de los demás (Moscovici 1986, citado en Covarrubias y Martínez 2007).

En el presente estudio, esperamos, por una parte, que las capacidades de la memoria espacial a corto plazo estén implicadas en el proceso de aprendizaje de una nueva pieza de música contemporánea; y por otra, que el desarrollo de una metodología basada en la problematización ${ }^{4}$ estimule las interacciones en la clase de piano y proporcione información significativa acerca del tipo de estrategias implementadas por los estudiantes de piano funcional en el aprendizaje de una nueva pieza de música. 


\section{METODOLOGÍA}

\section{DISEÑO}

El diseño de la investigación es mixto y emergente (Hernández, Fernández-Collado, y Baptista 2008). En efecto, se convierten datos cualitativos en cuantitativos a través de frecuencias. Se usa, tal como lo describe Flick (2012), un muestreo estadístico debido a que el tamaño de la muestra es conocido con antelación. Los datos cualitativos se obtienen de los cuestionarios realizados a los estudiantes y el profesor. Por su parte, los datos cuantitativos se obtienen de los resultados de la performance (tempo y errores), de las pruebas de memoria operativa y de memoria espacial a corto plazo, así como de las calificaciones del profesor a lo largo del año. En un primer nivel, se analizan las representaciones subjetivas de los estudiantes en relación con su propio proceso de aprendizaje y se contrastan con la visión del profesor. Se incluye información que da cuenta de la propia visión de los estudiantes acerca de la transmisión del conocimiento musical. En un segundo nivel, se correlacionan las diferentes variables estudiadas por medio de un análisis de componentes principales, donde las variables cualitativas convertidas en frecuencias son introducidas como variables suplementarias y no activas. Lo anterior con la finalidad de no modificar el baricentro en relación con las medidas llamadas puras.

Las técnicas o los instrumentos de toma de información son los cuestionarios, los registros de la performance al piano en formato MIDI y las pruebas de competencias cognitivas: prueba de amplitud lectora y Corsi block-tapping test.

Es necesario señalar que, en el contexto de estudio, parte importante del conocimiento teórico y práctico necesario para conocer y abordar los distintos estilos musicales se realiza a través de la asignatura de Piano Funcional. Esta ocupa, entonces, un rol transversal en la formación musical, más aún si consideramos que gran parte de los estudiantes entran en la Licenciatura en Música sin tener conocimientos previos en lectura musical (al menos de manera operativa). Muchos, por tanto, desconocen los rasgos estilísticos de cada periodo histórico y han de abordarlos por primera vez en la clase de piano. Un espacio dentro de ese repertorio está destinado al estudio de pianistas chilenos, principalmente a través de obras compuestas con objetivo pedagógico, muchas de ellas contemporáneas y con una marcada tendencia atonal (no siempre de vanguardia). Es en esta clase donde se estudian las particularidades metodológicas que exploraremos en el siguiente trabajo, aquellas propias del repertorio señalado.

\section{PARTICIPANTES}

Un grupo de diez pianistas de la Licenciatura en Música de la Facultad de Artes de la Universidad de Chile, todos alumnos de un mismo profesor, participaron en el experimento. Los participantes están entre el tercer y quinto año de esta licenciatura. Decidieron participar voluntariamente en la experiencia junto con su profesor y fueron informados acerca de los alcances de la investigación. Las variables de caracterización de grupo son presentadas en la tabla 1. A pesar de que algunos pianistas tenían experiencia previa con el piano antes de ingresar a esta licenciatura, todos fueron nivelados en un curso común (de nivel inicial) 
en lectura musical. Por lo anterior, si bien el rango de experiencia musical con el piano es variado, no ocurre así con la experiencia en lectura musical que es más bien homogéneo. Como lo habíamos comentado, esto es un caso bastante recurrente en la realidad formativa chilena. En efecto, solo dos de los diez pianistas iniciaron el aprendizaje de la lectura musical en el momento de iniciar el estudio del piano. Los otros participantes iniciaron los cursos de solfeo al ingresar a la universidad.

TABLA 1. VARIABLES DE CARACTERIZACIÓN (EN PARÉNTESIS LOS VALORES MEDIOS)

\begin{tabular}{|c|c|c|c|c|}
\hline N. ${ }^{\circ}$ de pianistas & Edad & Nivel & Experiencia & Práctica \\
\hline 10 & $21-26(23.4)$ & $3 . e r$ a $5 .^{\circ}$ año & $3-11$ años (6.1) & $60-180$ min (88.1) \\
\hline
\end{tabular}

Fuente: Elaboración propia.

\section{MATERIAL MUSICAL}

Corresponde a la pieza musical Algunas preguntas del compositor chileno Juan Amenábar. Es una pieza atonal sencilla de carácter íntimo. La obra está estructurada en forma de mosaico (i. e. variación continua de motivos rítmicos) y presenta una expansión en pulsaciones (beats) que se incrementa en la sección central (compases 7-12), para luego estrecharse en la última sección (compases 13-18). Cabe señalar que este tipo de forma es comparable a la moment form que caracteriza las composiciones de Stockhausen, uno de los compositores que influencia, sin duda, los trabajos de Amenábar, quien fuera pionero de la música electroacústica en Chile. La pieza fue creada por encargo de la Facultad de Artes de la Universidad de Chile, probablemente con la intención de difundir la música contemporánea chilena a pianistas principiantes. Técnicamente, la pieza no presenta grandes dificultades y se evidencia su carácter pedagógico.

\section{PRUEBAS DE MEMORIA}

Se aplica una prueba de amplitud lectora que corresponde a la segunda versión de Daneman y Carpenter (1980), la cual fue traducida desde el francés y administrada en una computadora. Los participantes debían leer frases breves y al mismo tiempo memorizar un número. Una vez leídas las frases, para pasar a la siguiente, debían decir si la frase era coherente o no. El número de frases por secuencia incrementa a medida que avanza la prueba. Se aplica, igualmente, una prueba de memoria espacial: el Corsi block-tapping test que consiste en reproducir ordenadamente una secuencia de figuras (bloques) tras verla presentada en la pantalla. El número de figuras por secuencia aumenta progresivamente hasta alcanzar el límite de las capacidades de los participantes. El test fue administrado de manera ascendente, es decir, las nueve figuras fueron presentadas en secuencias de tres intentos cada una. 


\section{PROTOCOLOS VERBALES}

Para tener acceso a las estrategias de toma, procesamiento y recuperación de la información musical utilizadas por los participantes durante el aprendizaje de una nueva pieza de música contemporánea, utilizaremos cuestionarios y la técnica de protocolos verbales retrospectivos.

Los protocolos verbales permiten obtener información en tiempo real o de forma retrospectiva sobre las estrategias y los pensamientos que preceden o acompañan a la acción. Estos pensamientos "articulados" pueden grabarse o anotarse, para, a continuación, ser analizados de acuerdo con las categorías definidas (Armengol 2007). Los protocolos verbales conducen a los sujetos a explicar sus pensamientos $y$, por tanto, permiten tener información sobre los procesos cognitivos, implicados en actividades como la solución de problemas o situaciones donde sea necesario acercarse al componente social y contextual del pensamiento (Ericsson y Simon 1980). En efecto, los autores entienden el pensamiento como una secuencia de estados cognitivos relativamente estables, por lo cual es importante que el investigador no influya en los pensamientos del participante.

\section{EQUIPOS Y PROCEDIMIENTO}

Los pianistas ejecutan la pieza en un teclado Roland HP 335 conectado a una interfaz Digidesign M Box que envía los datos a un ordenador portátil. Para el registro de datos en formato MIDI, se utilizó el software Cubase SX4. El pianista recibe un retorno audio a través de altavoces que se ubican al costado del teclado. Específicamente, se les pidió a los participantes leer a primera vista Algunas preguntas y, luego de dos minutos de práctica, volver a leer el trozo. Se repite el mismo esquema hasta completar cuatro interpretaciones sin detención de la pieza completa con sus correspondientes tres fases de práctica. Una vez finalizada la prueba de lectura, los pianistas realizan las pruebas de memoria operativa y de memoria espacial. Posteriormente, los pianistas, el profesor y los estudiantes profesores ${ }^{5}$ responden a un cuestionario. Se le pide al profesor, en una sesión suplementaria, evaluar las performances de sus alumnos mientras observa un video registrado el día del experimento.

Un primer nivel de análisis se basa en los cuestionarios para los estudiantes. El análisis de las unidades de sentido permite construir categorías que reflejen objetivamente el concepto del aprendizaje en interacción y, al mismo tiempo, describan las representaciones subjetivas que construyen los estudiantes de su propio aprendizaje.

Un segundo nivel de análisis se centra en los conceptos de base en la enseñanza del piano, la metodología y la evaluación (i. e. frecuencia, herramientas, parámetros y retroalimentación a los estudiantes) y en los objetivos específicos esperados por el profesor en la clase. Este análisis considera el punto de vista del profesor como también de los cuatro estudiantes profesores.

La evaluación de la performance y de los aspectos cualitativos se aborda utilizando un enfoque integrado. ${ }^{6}$ En general, el objetivo es identificar el tipo de representación mental involucrada en ella y obtener una información más rica sobre el proceso de aprendizaje y la interacción en el aula. Lo anterior con el objetivo de definir algunos indicadores clave para estudiar el aprendizaje a corto plazo y así dar cuenta de aprendizajes significativos que intervienen en un contexto dado. 


\section{RESULTADOS}

\section{CUESTIONARIOS A LOS ESTUDIANTES}

El análisis de los cuestionarios nos ha permitido tener acceso a las representaciones subjetivas de los estudiantes sobre su propia manera de aprender. Las preguntas estaban destinadas a identificar el tipo de estrategia utilizada por los pianistas (i. e. ¿cómo trabajó la pieza?) y a observar las capacidades metacognitivas (i. e. ¿cómo se dio cuenta de su progreso?). Los distintos indicadores fueron agrupados en tres categorías: 1) imitación (modelo del profesor), 2) técnica (conocimiento) y 3) autonomía (construcción del conocimiento), teniendo en cuenta que la adquisición de competencias permitirá finalmente a los pianistas ser autónomos.

No se observaron diferencias significativas entre las tres categorías. Sin embargo, los pianistas que declaran ser más autónomos parecen estar menos implicados en el aprendizaje imitativo $(r(8)=-0.66, p<0.05)$, o viceversa. En este caso, cabe preguntarse si la técnica representa una etapa intermedia entre el aprendizaje por imitación y la adquisición de la autonomía (véase tabla 2).

\section{TABLA 2. EJEMPLOS Y PORCENTAJES DE REPRESENTACIÓN DE LAS UNIDADES DE SENTIDO (AFIRMACIONES) SEGÚN LA CLASIFICACIÓN EN TRES CATEGORÍAS: IMITACIÓN, TÉCNICA Y AUTONOMÍA.}

\begin{tabular}{|c|c|c|}
\hline Categorías & $\%$ & Ejemplos de unidades de sentido \\
\hline Imitación & 29.49 & $\begin{array}{l}\text { Al principio, traté de leer verticalmente nota por nota sin tener } \\
\text { un buen resultado. }\end{array}$ \\
\hline Técnica & 38.46 & $\begin{array}{l}\text { Pulsación lenta... observar registro y en relación con esos } \\
\text { elementos encontrar la posición de las manos. }\end{array}$ \\
\hline Automomía & 32.05 & $\begin{array}{l}\text { Según la confianza que iba adquiriendo, pude tocar cada vez } \\
\text { más rápido sin cometer errores. }\end{array}$ \\
\hline
\end{tabular}

Fuente: Elaboración propia.

\section{CONCEPTOS DE BASE Y METODOLOGÍA}

El modelo evaluativo propuesto en el presente estudio nos ha permitido analizar en detalle las interacciones dentro de la clase de piano. En lo que respecta al contexto experimental, este está definido sobre tres niveles de interacción en la clase: 1) el profesor, 2) los estudiantes profesores y 3 ) los estudiantes no profesores.

Se le pide al profesor y a los estudiantes profesores que respondan a las siguientes preguntas: ¿cuáles son los conceptos de base que usted enseña? y ¿qué tipo de metodología utiliza?

Si comparamos los conceptos de base y las metodologías de enseñanza utilizadas por el profesor y los estudiantes profesores, se observa con rapidez que la estructura de la clase es prácticamente idéntica (i. e. organización del tiempo y procedimiento). Esto es lógico, ya que se trata de una clase de metodología pianística realizada por el mismo profesor a sus estudiantes 
profesores. Sin embargo, los conceptos básicos evaluados por ellos no siempre son los mismos que los de su profesora. Esto sugiere que los estudiantes profesores han ido elaborando a lo largo de la licenciatura una visión personal y creativa, es decir, una concepción de la transmisión del conocimiento musical más amplia (véanse tabla 3 y figura 1).

\section{TABLA 3. RESPUESTAS DE LOS ESTUDIANTES PROFESORES (EP) Y DEL PROFESOR (P) EN LO QUE RESPECTA A LOS CONCEPTOS DE BASE POR SER EVALUADOS.}

\begin{tabular}{|c|c|}
\cline { 2 - 3 } \multicolumn{1}{c|}{} & \multicolumn{2}{c|}{ ¿Cuáles son los conceptos de base que usted enseña? } \\
\hline P & La respiración, la relajación y la posición. \\
\hline EP 1 & $\begin{array}{l}\text { La utilización del cuerpo en relación con la sonoridad del piano y una buena compren- } \\
\text { sión (interpretación) de lo que aparece en la partitura. }\end{array}$ \\
\hline EP 2 & $\begin{array}{l}\text { Que aprendan a leer correctamente lo que pide la partitura, que tomen la responsabili- } \\
\text { dad de aquello que estudian, que logren hacer música y se diviertan tocando. }\end{array}$ \\
\hline EP 3 & La confianza, la creatividad, el respeto mutuo y el amor por lo que se hace. \\
\hline
\end{tabular}

Fuente: Elaboración propia.

\section{FIGURA 1}

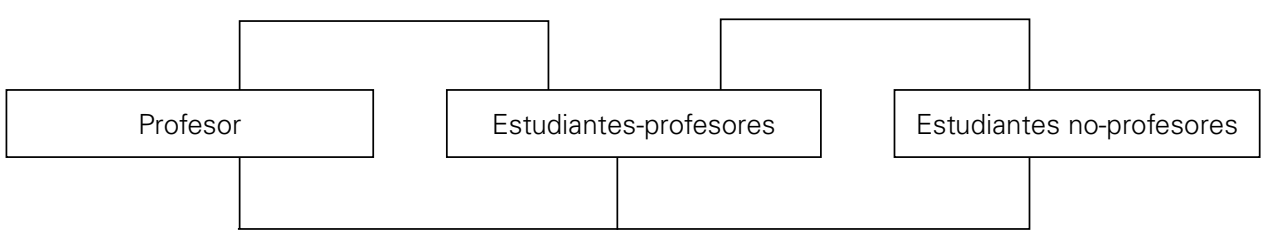

Figura 1. Contexto experimental. Se observa la existencia de dos flujos: el primero (abajo) representa el flujo habitual de la clase, definido por una estructura común; y el segundo (arriba), el flujo de los objetivos metodológicos.

Las cuatro interpretaciones de la misma pieza tenían como objetivo estimular la problematización ${ }^{7}$ y observar la evolución del rendimiento, con el fin de estudiar las estrategias de aprendizaje musical a corto plazo. Quisimos describir en un triángulo dialéctico las interacciones entre profesor y alumno y el papel de estos indicadores como coadyuvantes en el proceso de aprendizaje (véase figura 2).

Al mismo tiempo que los estudiantes buscan la eficacia en su interpretación, la tarea de la profesora es darles confianza. La implementación de un proceso continuo parece estar determinada por la capacidad de la profesora de detectar el potencial del estudiante y adaptar sus estrategias de enseñanza. En efecto, el análisis de un contexto dado es inherente al acto de enseñanza (Coll 1988). "Usar lo que tenemos nos da confianza [...] a largo plazo, el aprendizaje se consolida a través de un eje de potencialidad: cognitivo/motor y motor/cognitiva" (Kenya Godoy, comunicación personal). De lo anterior se desprende que el rol del profesor es dar confianza al estudiante para conducirlo a ser autónomo en su proceso de aprendizaje. La autonomía del estudiante se refiere aquí claramente a un objetivo educativo importante. 
FIGURA 2

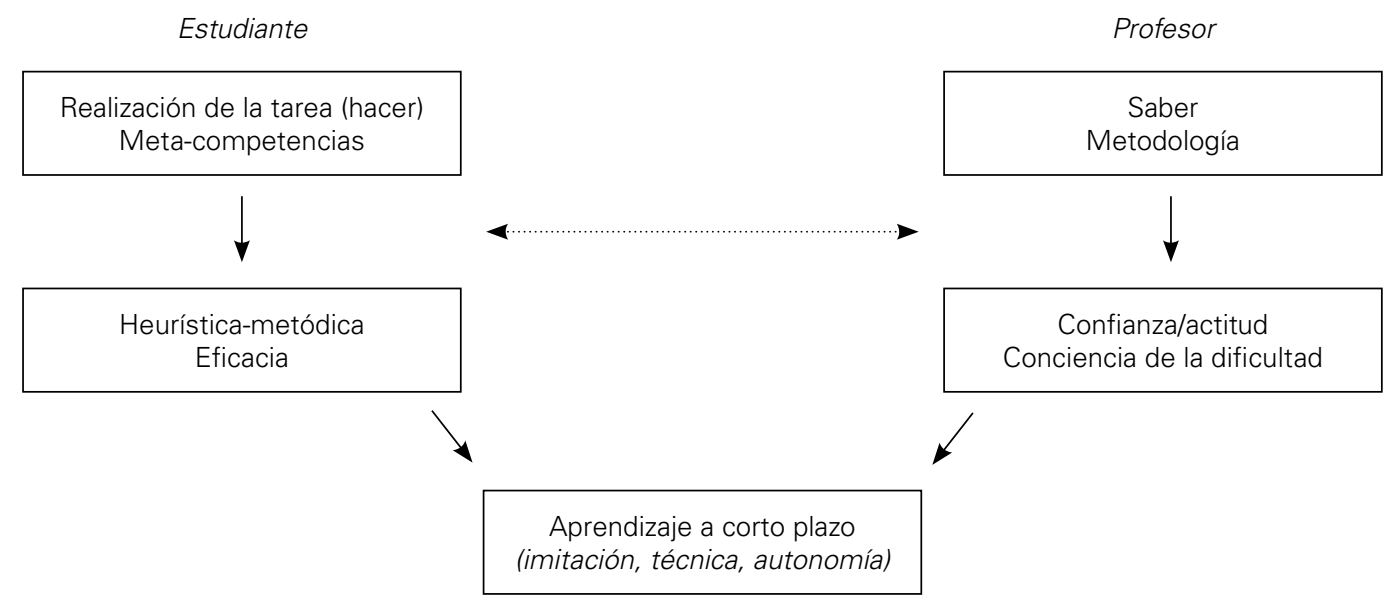

Figura 2. Esquema que representa la relación entre la actividad del maestro (a la derecha) y la del estudiante (a la izquierda). El estudiante realiza la tarea (p. ej. aprender una nueva pieza) y busca ser eficaz (p. ej. cuidando aspectos técnico-musicales), manteniendo su musicalidad. El estudiante pasa progresivamente de un comportamiento heurístico adaptativo a un comportamiento basado en objetivos personales. De esta manera, las metacompetencias de los estudiantes se actualizan y el maestro con su información (saber) ayuda al estudiante a reafirmar su confianza personal, entregándole los medios para tomar conciencia de sus dificultades.

\section{PARTICULARIDADES METODOLÓGICAS DEL PROCESO DE LECTURA A PRIMERA VISTA}

Según la opinión de Kenya Godoy, la asignatura de Piano Funcional consiste en desarrollar un manejo técnico que prepare al estudiante para mantener la parte histórica del repertorio (desde el Barroco hasta lo contemporáneo). Desde un punto de vista teórico, lo anterior se podría conseguir 1) a través del conocimiento de la notación musical, 2) logrando leer música de manera fluida y en un pulso tranquilo y 3) ejecutando de manera coherente la partitura a medida que se va integrando la información musical. Además, el profesor comenta que en la lectura a primera vista es necesario saber identificar la tonalidad, pensar en un pulso lento y poner especial atención a las figuras rítmicas. En la partitura de música clásica, sería indispensable el trabajo con la armonía tradicional, tener claro el ritmo armónico y conocer las funciones armónicas. Desde un punto de vista psicológico, implicaría hacer hincapié en los marcadores estructurales ( $p$. ej. cadencias, finales de frases, ritmo armónico), para poder aumentar la anticipación dada la naturaleza secuencial del discurso. En la partitura de música romántica, en cambio, si bien cuentan los mismos elementos, el tratamiento de la lectura a primera vista estaría estrechamente ligado al compositor. Lo más característico en este periodo, según Kenya Godoy, es el uso de la armonía (marcadores estructurales); por tanto, es necesario "entender al compositor". En la música contemporánea, muchas partituras contienen una simbología que se debe respetar, que es específica para cada obra. Frecuentemente, un mismo compositor utiliza un tipo de simbología similar en varias de sus obras. Básicamente, se trata de tener experiencia en distintos tipos de notación; por tanto, la lectura a primera vista debería estar en manos de un pianista especializado. En efecto, desde la música romántica hasta nuestros días, el trabajo de lectura a primera vista requiere una especialización cada vez mayor. Lo anterior se ve reflejado en el trabajo especializado que realizan muchos panistas acompañantes. 


\section{ANÁLISIS DE COMPONENTES PRINCIPALES}

Con la finalidad de correlacionar el conjunto de variables estudiadas y analizar particularmente la relación de estas con las competencias cognitivas (pruebas de memoria operativa y espacial a corto plazo), se realizó un análisis de componentes principales. Se incluyeron las variables de la performance, los resultados obtenidos en las pruebas de memoria operativa verbal y memoria espacial, la evaluación del profesor y las variables cualitativas provenientes del análisis de los cuestionarios (frecuencia de observación de cada indicador) que se incluyeron como variables suplementarias (Husson, Lê y Pagès 2009). Se utilizaron las llamadas categorías puras y no reagrupadas (i. e. imitación y autonomía), aquellas que obtuvieron el mayor porcentaje de representación. Los análisis consideran los puntajes globales de tempo y errores (valor medio de los cuatro ensayos).

En cuanto a las variables de la performance, se observa que son independientes, en las cuales se destaca una fuerte correlación entre el tempo y los conceptos de base evaluados por el profesor (i. e. respiración, relajación y posición). La independencia entre estos y los errores sugiere que son pertinentes para evaluar el proceso y no solo los resultados (véase figura 3).

\section{FIGURA 3}

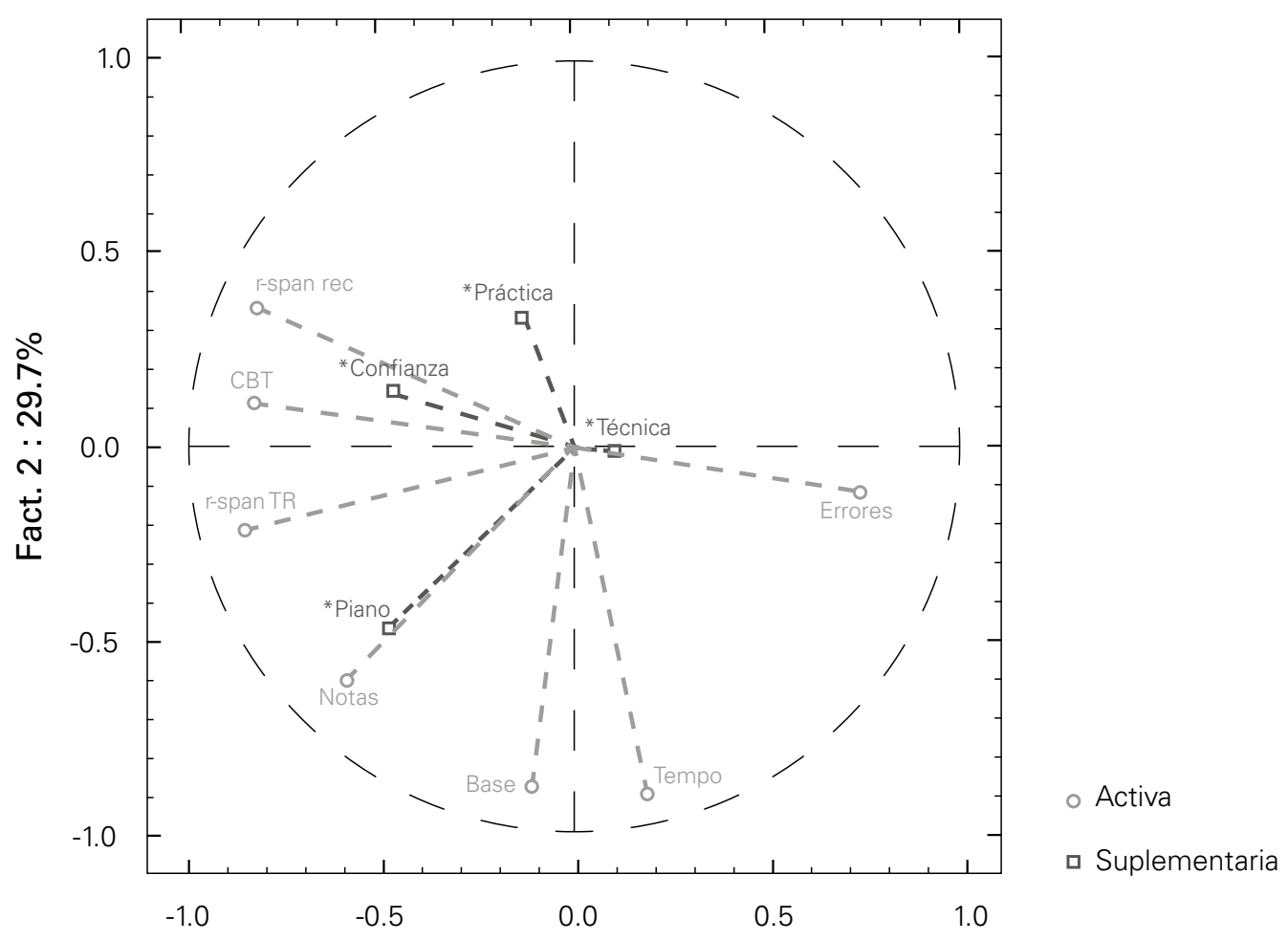

Fact. $1: 42.78 \%$

Figura 3. Conjunto de variables estudiadas en el plano factorial. Los resultados de las pruebas de memoria operativa: tiempo de reacción (r-span TR), capacidad de almacenamiento (r-span rec), memoria espacial (CBT), variables de la performance (tempo y errores), evaluaciones del profesor -notas a lo largo del año (Notas) - y evaluación de los conceptos de base (Base). En las variables suplementarias, se incluyen las variables de caracterización: número de años de práctica de piano (Piano), el número de horas de práctica por día (Práctica) y las variables cualitativas provenientes de los

cuestionarios: afirmaciones que implican un componente técnico (Técnica) y afirmaciones que implican un componente actitudinal (Confianza). 
Como se observa en el análisis de componentes principales, la memoria espacial está correlacionada con la memoria verbal, probablemente debido a un efecto de contexto. La notación del profesor se encuentra en el mismo eje de los recursos cognitivos y sería un compromiso entre estas capacidades y los resultados de la performance, lo cual sugiere la existencia de una línea imaginaria que separa los dos ejes factoriales a partir de esta variable (i. e. notas). Se observa que las variables suplementarias, provenientes de los cuestionarios (i. e. técnica y confianza), están tendencial y negativamente correlacionadas, además de débilmente representadas en el plano factorial.

Del mismo modo, se observa una correlación tendencial entre los años de piano y los errores $(p=0.06)$. Para verificar esta observación, se realiza una regresión que muestra que los errores y el tempo tienen un impacto en los criterios básicos evaluados por el profesor $\left(R^{2}=44 \%, p<0.05\right)$, pero no en su calificación a lo largo del año.

\section{DISCUSIÓN}

Se estudia el proceso de aprendizaje de una nueva pieza de música contemporánea en una clase de piano utilizando un diseño de investigación mixto. Hemos abordado la calidad de la interacción, las concepciones metodológicas de los actores y su visión de la transmisión del conocimiento musical. Las cuatro ejecuciones de la misma pieza y las tres fases de práctica estaban dirigidas a estimular la problematización. Lo anterior nos permitió analizar el proceso de aprendizaje y observar la evolución de las ejecuciones pianísticas.

La presencia de tres categorías (i. e. imitación, autonomía y técnica) ha permitido observar un vínculo entre el aprendizaje por imitación y la adquisición de la autonomía. Esto es congruente con los resultados de Faller y Gruhn (2002), para quienes el aprendizaje determina un proceso que va de la imitación a la anticipación. Los autores reportan tiempos de reacción más eficientes en aquellos participantes que lograban tener una mejor representación mental de la música al realizar una tarea de repetición de patrones rítmicos y melódicos. La autonomía parece ser, entonces, un buen indicador de la ocurrencia de procesos cognitivos de alto nivel, ya que, tal como lo sugiere Gruhn (2004), se relaciona con la integración y la aplicación de los conocimientos. En igualdad de condiciones, las metodologías problematizadoras, centradas en mecanismos procedurales, serían pertinentes para evaluar el nivel de autonomía de los estudiantes. De esto, la anticipación y la autonomía serían buenos indicadores de la adquisición de competencias.

Aprender a aprender es orientarse hacia la propia transformación del individuo como aprendiz en diferentes contextos. Aprender a aprender es, por tanto, prepararse para ser autónomos (Portine 1998, 77).

El papel de las metacompetencias, ${ }^{8}$ particularmente la dimensión en la cual es posible aplicar lo aprendido y lograr realizar con éxito la tarea, aparece como un vector de confianza. Esto es consistente con Kermarrec (2004), para quien construir, recuperar y asociar los conocimientos permite la comprensión de la situación, la planificación y el control de la acción, lo cual forma parte del proceso de adquisición de competencias (17). ${ }^{9}$ Esto no excluye, a nuestro entender, que la búsqueda de la eficiencia en la performance pase a través de un comportamiento heurístico, ya que, tal como lo plantea Drake y Palmer (2000), la experiencia tiene un papel significativo en la planificación de la lectura como en los procesos de anticipación. Por lo demás, desde un paradigma constructivista, las nuevas adquisiciones dependen del conocimiento previo y representan un puente hacia nuevos aprendizajes. 
Analizando los cuestionarios de los estudiantes profesores, se observa que presentan un comportamiento similar a su profesor en la manera en que organizan la estructura de la clase. Sin embargo, en lo que respecta a los criterios de evaluación (objetivos esperados), el comportamiento es personal. Se puede inferir, como una primera posibilidad, que una identidad musical se encuentra en construcción, lo cual se traduce en un proceso de actualización de las metacompetencias. Este proceso de construcción requerirá una renegociación en la transición desde la vida de estudiante hasta que este deba enfrentar el mercado laboral (Juuti y Littleton 2012). Esto sugiere, igualmente, la existencia de un vínculo con el proceso de profesionalización, ${ }^{10}$ que se manifiesta en la adecuación a un lenguaje académico y una lógica institucional. Una segunda posibilidad podría estar basada en la transmisión metodológica entre el profesor y sus estudiantes, donde hemos mencionado la importancia del compromiso personal (Maturana 2002). Sin este sentido de pertenencia, el conocimiento no se puede transmitir.

Destacamos, desde el punto de vista metodológico, la importancia que le atribuye Kenya Godoy al desarrollo del trabajo de lectura analítica antes de la interpretación. Esta práctica permitiría integrar una serie de elementos dentro de los cuales podemos destacar el reconocimiento de aquellas secciones de la partitura donde hay dificultad, el tempo de ejecución, las alturas, los cambios de llave, los desplazamientos, la rítmica y el fraseo, entre otros. En relación con el contexto de estudio, esta metodología resulta ser transversal para todos los repertorios estudiados, lo cual constituye una puerta de entrada para que el estudiante, desde su propia visión del conocimiento, y según su propia experiencia, construya su propio saber. Lo anterior se hace más evidente en los flujos metodológicos, donde se observa que ya tempranamente comienzan a desarrollarse modelos propios de evaluación.

\section{CONFIANZA PARA EL APRENDIZAJE}

El análisis de las verbalizaciones muestra que los estudiantes adquieren confianza gradualmente a medida que realizan la tarea de lectura. La confianza facilita el aprendizaje y crea una dinámica entre el profesor y el estudiante, favorable para la adquisición de competencias a largo plazo (Rousseau, Deslandes y Fournier 2009). Esto es consistente con McPherson y McCormick (2006) para quienes la autoeficacia es un predictor de éxito, en aquellos estudiantes de música que rinden exámenes de desempeño instrumental de gran dificultad. Desde esta perspectiva, el que es capaz de integrar nuevas ideas e incorporarlas en su acerbo, por un lado, puede entender cómo aprendió y a su vez manifiesta su confianza en aquello que ha conseguido. La confianza cristaliza el aprendizaje a corto plazo y permite que el aprendizaje sea significativo. "En el momento de tocar, se revela su potencialidad, entonces, es necesario trabajar desde este potencial" (Kenya Godoy, comunicación personal).

A través del foco cualitativo de este estudio, hemos podido obtener mayor información respecto de los procesos creativos que permiten al estudiante replantearse y reorganizar el conocimiento. En efecto, creemos que la interacción creativa en la clase de piano favorece el aprendizaje. Como hemos visto, la confianza en sus diferentes dimensiones tiene un efecto sobre el rendimiento del estudiante y, posiblemente, en la adquisición de capacidades cognitivas generales. ${ }^{11}$ Lo anterior se observa en el análisis de componentes principales donde las pruebas de memoria y el indicador de confianza se agrupan en un mismo eje. 
Las capacidades cognitivas generales, particularmente la capacidad de la memoria operativa verbal, se asocia con performances más precisas en el piano (menos errores) y, a su vez, con las competencias requeridas por la clase de piano (notas del año). Por otra parte, los años de experiencia pianística se correlacionan, asimismo, con el eje de las capacidades de la memoria operativa, lo cual podría interpretarse como un efecto de transferencia de la práctica musical hacia las capacidades cognitivas de base. Sin embargo, dada la naturaleza teórica de esta asociación, debemos ser prudentes al sugerir que existe una relación de causalidad.

En cuanto a la evaluación de la profesora (criterios de base), en otras palabras, lo que ella espera de sus estudiantes, esta aparece ligada con la velocidad de interpretación de la pieza (tempo), lo cual es consistente con la literatura en cuanto al índice de adquisición de competencias (Drake y Palmer 2000). La regresión efectuada muestra que la precisión (tempo y errores) también tendría un impacto en la evaluación inicial, pero no en las notas a lo largo del año. Esto confirma que el profesor evalúa positivamente a sus estudiantes respecto del proceso y no solo en relación con la experiencia previa. Sin embargo, si el resultado no es el esperado (p. ej. dos pianistas que no cumplieron con las expectativas de Kenya Godoy), la evaluación del profesor tiende a centrarse en los errores. Estos resultados podrían complementar la información planteada en el triángulo dialéctico, particularmente lo referido a la relación entre la eficiencia y la evaluación de la profesora. En otras palabras, la eficacia buscada por el estudiante es compensada por una buena evaluación, lo que pasa por la realización de la conducta requerida por la profesora (respiración, relajación y posición). Por su parte, la aplicación de estos conceptos de base permite a los estudiantes ser más eficientes en su interpretación.

Lo anterior nos hace cuestionarnos sobre el vínculo entre la confianza personal y la capacidad de la memoria operativa. Según Baddeley (2000), el conocimiento cristalizado tiene una influencia indirecta sobre las capacidades de la inteligencia fluida. Citemos la siguiente verbalización de Kenya Godoy: "Estando tensa y ansiosa, ella toma más tiempo del que necesita (para aprender), porque mentalmente no se concentra" (comunicación personal). En efecto, la realización de tareas que miden las capacidades de la memoria espacial involucra las funciones ejecutivas y el control de la atención (Miyake et al. 2001). Por otra parte, las capacidades de la memoria espacial en nuestro análisis de componentes principales están débilmente correlacionadas con los conceptos de base intencionados por el profesor; y por otra, la velocidad de ejecución (tempo) se opone a los errores. A su vez, los pianistas con mejores capacidades de memoria espacial a corto plazo no fueron más precisos en su ejecución. Esto confirma nuestra observación inicial acerca de la tendencia del profesor a generalizar los criterios de evaluación en relación con los aspectos de base si el estudiante no logra los objetivos esperados. Esta tendencia a globalizar los criterios de evaluación reposa sobre la apreciación (negativa) respecto de las capacidades atencionales y de concentración de los estudiantes. ¿El profesor podría entonces tener una incidencia sobre los componentes fluidos de la inteligencia para construir una nueva forma de aprender?

Hemos observado una baja presencia de indicadores de la modalidad visual en las verbalizaciones. Esto es congruente con los resultados de Williamon, Valentine y Valentine (2002), quienes observan que, en los protocolos verbales que no comprometen la ejecución instrumental, las representaciones visuales son menos recurrentes. Por el contrario, en los 
protocolos verbales de la profesora, la modalidad visual está mucho más presente, especialmente en relación con la forma en que los pianistas miran el teclado mientras ejecutan la pieza, la comprobación de errores y la postura. Esto también parece estar estrechamente relacionado con la manera particular que tiene el profesor de entender el proceso de aprendizaje. Desde la perspectiva de la teoría de la actividad y considerando lo postulado por Marchand (2009), la acción y la reflexión estarían reguladas en la interacción donde el profesor representa la posición académica. Nos enfrentamos, entonces, a una manera de seleccionar la información que promueve la adopción de un discurso académico que finalmente parece influir en las representaciones subjetivas de los estudiantes en cuanto a su propia manera de aprender.

Todas estas observaciones nos llevan a sugerir la existencia de un eje que representa las capacidades de la memoria y un segundo eje que describe los aspectos técnicos de la interpretación instrumental. El punto de fusión de estos dos ejes podría situarse entre la calificación del profesor y los años de experiencia pianística de los estudiantes. La interpretación de las variables suplementarias del análisis de componentes principales sugiere, por una parte, que la confianza personal se asocia positivamente con la adquisición de metacompetencias disciplinares, y por otra, que la performance centrada en los aspectos técnicos conlleva cometer más errores y no asegura el cumplimiento de los objetivos perseguidos.

\section{CONCLUSIÓN}

En síntesis, el grado de confianza que le otorga el profesor a su alumno genera una dinámica que conlleva reforzar las adquisiciones a largo plazo. Por otra parte, la notación del profesor sería un compromiso entre la evaluación de las capacidades cognitivas (componente de fluido) y el aprendizaje del año (componente cristalizado). El proceso de aprendizaje se caracteriza por el paso de una fase imitativa a una fase autónoma donde las habilidades técnicas son una etapa intermedia, lo cual es congruente con la bibliografía consultada (Fitts 1964; Gruhn 2004). El profesor tendría una influencia en la metacognición de los estudiantes. Desde esta perspectiva, la adquisición de la autonomía es un indicador del aprendizaje a corto plazo. En definitiva, podemos afirmar que en el presente trabajo los enfoques cuantitativos y cualitativos han sido pertinentes y se refieren a fenómenos comunes. 


\section{NOTAS}

1 La concepción de música contemporánea no es exhaustiva en este trabajo. Ella representa, respecto de notación musical, un modelo similar al de la música clásico-romántica.

2 La especialización estructural de la corteza cerebral depende del mantenimiento de sinapsis neuronales preferentes (composición anatómica). Por su parte, la plasticidad funcional tiene relación con la interconectividad de las zonas corticales primarias del cerebro (transmisión de información).

3 El modelo da cuenta de la forma en que los expertos organizan su conocimiento según estructuras estables a través de las cuales pueden recuperar información relevante desde la memoria a largo plazo. Lo anterior permitiría sobrepasar las capacidades de la memoria a corto plazo y gestionar eficazmente las altas demandas de información que se requiere para realizar determinadas tareas específicas como la lectura a primera vista.

4 Esta metodología es conocida como coadyuvante a diversas actividades que pueden realizarse en un marco curricular de educación musical. Por otra parte, existe evidencia científica de que el uso de esta metodología podría ir en detrimento de la adquisición de esquemas mentales (Sweller 1988). En nuestro estudio, la problematización decanta en la movilización de diferentes estrategias que permiten resolver los problemas técnicos e interpretativos asociados al aprendizaje de una nueva pieza de música contemporánea. Lo anterior dentro de ciertos límites temporales (una corta sesión de aproximadamente $30 \mathrm{~min}$ ) y según una estructura de práctica musical (2 min entre cada ejecución) requerida por el investigador.

5 Entre los alumnos de la clase, tres de ellos eligieron, en su último año de estudios, seguir un curso de metodología pianística que realizan con su mismo maestro de piano.

6 Conceptualmente, esto implica, en lugar de un enfoque polarizado, la aplicación de un enfoque "continuo", que sitúa los conocimientos según su nivel de inferencia en una escala común. De esta manera, el conocimiento se organiza en dimensiones transversales a los métodos cuantitativos y cualitativos (Ercikan y Roth 2006).

7 Se adhiere a la visión de Marchand (2009), para quien la problematización no necesariamente tiene como objetivo alcanzar un aprendizaje específico, sino más bien problematizar una situación musical.

8 Las competencias se refieren, por ejemplo, a las capacidades prácticas; en cambio, las metacompetencias designan el conocimiento de los métodos para la adquisición de dichas capacidades.

9 Al respecto, véase Marteniuk (1986) y Richard (1998).

10 La reflexión sobre los procesos de profesionalización se ha centrado particularmente, según Chapoulie (1973), en los sistemas de legitimación que sustentan la creencia colectiva según la cual una ocupación debe ser entendida como una profesión. La adquisición de este estatus es codiciado en la medida en que concede ciertos privilegios (Danner y Galodé 2009, 7).

11 En efecto, el componente emocional del aprendizaje, relacionado con el desarrollo de la memoria operativa, ha sido poco estudiado. Podemos citar una investigación que estudia la relación entre la memoria operativa y el mindfulness-training (Jha et al. 2010) y otra que remarca el efecto de la información emocional negativa sobre la memoria operativa y la memoria a largo plazo (Kensinger y Corkin 2003).

\section{REFERENCIAS}

Anguera, Joaquin A., Patricia A. Reuter-Lorenz, Daniel T. Willingham y Rachael D. Seidler. 2010. "Contributions of Spatial Working Memory to Visuomotor Learning". Journal of Cognitive Neuroscience 22 (9): 1917-1930.

Armengol Castells, Lurdes. 2007. "Los protocolos de pensamiento en voz alta como instrumento para analizar el proceso de escritura". Revista Española de Lingüística Aplicada 20: 27-35.

Avendaño, Cecilia, Mariane Krause y María Inés Winkler. 1993. “Representaciones sociales y teorías subjetivas: relevancia teórica y aplicaciones empíricas". Psykhe 2 (1): 107-114. 
Baddeley, Alan. 2000. "The Episodic Buffer: A New Component of Working Memory?". Trends in Cognitive Sciences 4 (11): 417-423.

Baddeley, Alan. 2012. "Working Memory: Theories, Models, and Controversies". Annual Review of Psychology 63: 1-29.

Baddeley, Alan y Hitch Graham. 1974. "Working Memory." En The Psychology of Learning and Motivation: Advances in Research and Theory, editado por Arthur C. Graesser y Gordon H. Bower, 47-89. Nueva York: Academic Press.

Berz, William L. 1995. "Working Memory in Music: A Theoretical Model". Music Perception: An Interdisciplinary Journal 12 (3): 353-364.

Bo, Jin y Rachael D. Seidler. 2009. “Visuospatial Working Memory Capacity Predicts the Organization of Acquired Explicit Motor Sequences". Journal of Neurophysiology 101 (6): 3116-3125.

Bogunovic, Blanka. 2003. How does teacher make a difference? En Proceedings of the 5th Triennial ESCOM Conference, editado por R. Kopiez, A. C. Lehmann, I. Wolther y C. Wolf, 282-284. Hanover: Hanover University of Music and Drama.

Bressoux, Pascal y Phillippe Dessus. 2003. "Stratégies de l'enseignant en situation d'interaction". En Les sciences cognitives et l'école: la question des apprentissages, editado por Michèle Kail y Michel Fayol, 213-257. París: Presses Universitaires de France.

Brochard, Renaud, Andre Dufour y Olivier Despres. 2004. "Effect of Musical Expertise on Visuospatial Abilities: Evidence from Reaction Times and Mental Imagery". Brain and Cognition 54 (2): 103-109.

Cara, Michel André. 2017. "Anticipation Awareness and Visual Monitoring in Reading Contemporary Music." Musicae Scientiae. http://journals.sagepub.com/doi/abs/10.1177/1029864916687601

Cara, Michel André y Gabriela Gómez. 2016. "Silent Reading of Music and Texts: Eye Movements and Integrative Reading Mechanisms". Journal of Eye Movement Research 9 (7): 1-17.

Coll, César. 1988. "Significado y sentido en el aprendizaje escolar: reflexiones en torno al concepto de aprendizaje significativo". Infancia y Aprendizaje 11 (41): 131-142.

Covarrubias Papahiu, Patricia y Claudia Cecilia Martínez Estrada. 2007. "Representaciones de estudiantes universitarios sobre el aprendizaje significativo y las condiciones que lo favorecen" Perfiles Educativos 29 (115): 49-71.

Chapoulie, Jean-Michel. 1973. "Sur l'analyse sociologique des groupes professionnels". Revue française de sociologie 14 (1) 86-114.

Daneman, Meredyth y Patricia A. Carpenter. 1980. "Individual Differences in Working Memory and Reading". Journal of Verbal Learning and Verbal Behavior 19 (4): 450-466.

Danner, Magali y Gilles Galodé. 2009. "Analyse des processus de fermeture dans l'accès à l'emploi: Les professions indépendantes vocationnelles". Les cahiers de I'IREDU 70.

Drake, Carolyn y Caroline Palmer. 2000. "Skill Acquisition in Music Performance: Relations between Planning and Temporal Control." Cognition 74 (1): 1-32.

Ercikan, Kadriye y Wolff-Michael Roth. 2006. "What Good Is Polarizing Research Into Qualitative and Quantitative?". Educational Researcher 35 (5): 14-23.

Ericsson, K. Anders y Herbert A. Simon. 1980. "Verbal Reports as Data". Psychological Review 87 (3): 215-251.

Ericsson, K. Anders, Ralf T. Krampe y Clemens Tesch-Römer. 1993. "The Role of Deliberate Practice in the Acquisition of Expert Performance". Psychological Review 100 (3): 363-406.

Ericsson, K. Anders y Walter Kintsch. 1995. "Long-term Working Memory". Psychological Review 102 (2): $211 .-245$.

Faller, Wolfgang y Wilfried Gruhn. Music Learning: From Imitation to Anticipation. Presentación en la 7th International Conference on Music Perception and Cognition, Causal Productions, Sídney, julio 23-28, 2002.

Fitts, Paul M. 1964. "Perceptual-Motor Skill Learning". En Categories of Human Learning, editado por Arthur, W. Melton, 243-285. Nueva York: Academic Press.

Flick, Uwe. 2012. Introducción a la investigación cualitativa. Madrid: Morata. 
Genthon, Michèle. 1997. Apprentissage-évaluation-recherche: genèse des interactions complexes comme ouvertures régulatrices. Marsella: Université de Provence, Département des sciences de l'éducation.

Gruhn, Wilfried. 2004. "Are Different Types of Mental Representation Reflected by Brain Activation Patterns." En Proceedings of the 8th International Conference on Music perception and Cognition, editado por S. D. Lipscomb, R. Ashley, R. O. Gjerdingen y P. Webster, 124-127. Evanston, IL.

Hernández Sampieri, Roberto, Carlos Fernández-Collado y María del Pilar Baptista Lucio. 2008. Metodología de la investigación. México: McGraw-Hill.

Husson, François, Sébastien Lê y Jérôme Pagès. 2009. Analyse de données avec R. Rennes: Presses Universitaires de Rennes.

Jha, Amishi P., Elizabeth A. Stanley, Anastasia, Kiyonaga, Ling Wong y Lois Gelfand, 2010. “Examining the Protective Effects of Mindfulness Training on Working Memory Capacity and Affective Experience". Emotion 10 (1): 54-64.

Juuti, Sini y Karen Littleton. 2012. "Tracing the Transition from Study to a Contemporary Creative Working Life: The Trajectories of Professional Musicians". Vocations and Learning 5 (1): 5-21.

Kensinger, Elizabeth A. y Suzanne Corkin. 2003. “Effect of Negative Emotional Content on Working Memory and Long-Term Memory". Emotion 3 (4): 378-393.

Kermarrec, Gilles. 2004. "Stratégies d'apprentissage et autoregulation: Revue de question dans le domaine des habiletés sportives". Movement \& Sport Sciences 3: 9-38.

Kopiez, Reinhard y Ji In Lee. 2008. "Towards a General Model of Skills Involved in Sight Reading Music". Music Education Research 10 (1): 41-62.

Marchand, Chrystel. 2009. Pour une didactique de l'art musical. París: L'Harmattan.

Maturana, Humberto. 2002. El sentido de lo humano. Santiago de Chile: Dolmen.

Marteniuk, Ronald G. 1986. "Information Processes in Movement Learning". Journal of Motor Behavior 18 (1): 55-75.

McAdams, Stephen. 1989. "Psychological Constraints on Form-Bearing Dimensions in Music". Contemporary Music Review 4 (1): 181-198.

McPherson, Gary E. y John McCormick. 2006. "Self-efficacy and music performance". Psychology of Music 34 (3): 322-336.

Meyer, Leonard B. 1956. Emotion and Meaning in Music. Chicago: University of Chicago Press.

Miyake, Akira, Naomi P. Friedman, David A. Rettinger, Priti Shah y Mary Hegarty. 2001. "How are Visuospatial Working Memory, Executive Functioning, and Spatial Abilities Related? A Latent-Variable Analysis". Journal of Experimental Psychology: General 130 (4): 621-640.

Moscovici, Serge. 1986. Psicología social II. Pensamiento y vida social. Psicología social y problemas sociales. Barcelona: Paidós.

Narmour, Eugene. 1990. The Analysis and Cognition of Basic Melodic Structures: The ImplicationRealization Model. Chicago: University of Chicago Press.

O'Sullivan, Tim et al. 1997. Conceptos clave en comunicación y estudios culturales. Buenos Aires: Amorrortu.

Pearce, Marcus T. y Geraint A. Wiggins. 2006. "Expectation in Melody: The Influence of Context and Learning". Music Perception: An Interdisciplinary Journal 23 (5): 377-405.

Portine, Henri. 1998. "L'autonomie de l'apprenant en questions". Apprentissage des Langues et Systèmes $d^{\prime}$ Information et de Communication 1 (1): 73-77.

Richard, Jean-François. 1998. Les activités mentales. París: Armand Colin.

Rizo García, Marta. 2006. "La interacción y la comunicación desde los enfoques de la psicología social y la sociología fenomenológica: breve exploración teórica." Anàlisi: quaderns de comunicació i cultura 33: 45-62.

Rousseau, Nadia, Rollande Deslandes y Hélène Fournier. 2009. "La relation de confiance maître-élève: perception d'élèves ayant des difficultés scolaires". McGill Journal of Education 44 (2): 193-211. 
Roux, Franck-Emmanuel et al. 2007. "When "Abegg" is Read and ("A, B, E, G, G") is not: A Cortical Stimulation Study of Musical Score Reading". Journal of Neurosurgery 106 (6): 1017-1027.

Sergent, J., E. Zuck, S. Terriah y B. MacDonald. 1992. “Distributed Neural Network Underlying Musical Sight-Reading and Keyboard Performance". Science 257 (5066): 106-109.

Servant, Isabelle y Thierry Baccino. 1999. "Lire Beethoven: une étude exploratoire des mouvements des yeux". Musicae Scientiae 3 (1): 67-94.

Sloboda, John A. 1982. "Music Performance". En Psychology of Music, editado por Diana Deutsch, 479496. Nueva York: Academic Press.

Stewart, Lauren, Rik Hensonab, Knut Kampea, Vincent Walsha, Robert Turnerb y Uta Fritha. 2003. "Brain Changes After Learning to Read and play Music." Neuroimage 20 (1): 71-83.

Sweller, John. 1988. "Cognitive Load During Problem Solving: Effects on Learning". Cognitive Science 12 (2): 257-285.

Williamon, Aaron, Elizabeth Valentine y John Valentine. 2002. "Shifting the Focus of Attention between Levels of Musical Structure". European Journal of Cognitive Psychology 14 (4): 493-520.

Wolf, Thomas. 1976. "A Cognitive Model of Musical Sight-Reading". Journal of Psycholinguistic Research 5 (2): 143-171.

\section{Cómo citar:}

Cara, Michel, A. 2018. “Representaciones subjetivas y competencias cognitivas en el aprendizaje de una nueva pieza de música contemporánea para piano". Cuadernos de Música, Artes Visuales y Artes Escénicas 13 (2): 205-226. http://doi.org/10.11144/javeriana.mavae13-2.rsyc 